\title{
Changing hospitals for a changing medicine
}

\author{
by Marco Confalonieri
}

The medical specialty dealing with respiratory diseases initially was devoted to a single disease, tuberculosis (TB), also named the white plague. Until the middle of last century, tuberculosis was so important epidemiologically (high incidence and mortality) that there were large hospitals completely dedicated to this disease. They were the sanatoriums which were built in places generally healthy, well exposed to the sun and surrounded by trees and greenery. The invention of the sanatorium dates back to an era when there were not valid pharmacological treatments against tuberculosis and were therefore emphasized the rich and healthy diet, resting, sun exposure and breathing "good air". The duration of hospital stay was usually such a long time that patients could stay in the sanatorium even years. Not by chance it has led to a literature of sanatoriums, whose greatest exponent was the Nobel Prize winner Thomas Mann in his book "The Magic Mountain". As director of a department of Pulmonology I have been called in Trieste in 2000 to work in one of the last sanatoriums then operating in Italy, soon would be closed and put to another use. The Sanatorium "Santorio Santorio" was built after the end of the second world war by the Americans who at that time had introduced streptomycin, the first bactericidal drug against the mycobacterium of tuberculosis. The Hospital Santorio of Trieste had up to 400 beds and six departments of pulmonology, but now is converted into a University Centre of Research, the SISSA (International School of Advanced Studies Superior), which specializes in neuroscience, mathematics, and theoretical physics (Figure 1). Shortly before the closing of the Sanatorium of Trieste, in 2000 there were still 70 beds for the pneumological Department admitting about 1200 patients per year. Now, after 14 years the Department of Pneumology is allocated at the University Hospital of Cattinara (Figure 2), built in 1984 with innovative technological solutions (it was then installed in Trieste, the first European PACS radiology). The Cattinara's Hospital is the general hospital for acute and complex patients, with a typical Hub characteristics (cardiosugery, neurosurgery, thoracic surgery, plastic surgery, interventional bronchology, pediatric and neonatal ICU, interventional cardiology 24hrs, nuclear medicine, and so on). The Department of Pneumology has got now only 17 beds, but the number of admission per year is the same than in 2000, for the reason that there is now a Respiratory Intensive Care Unit (RICU) with increased turn-over and a lower hospital stay. Moreover, most specialized pneumological activities are now performed as dayhospital care and outclinics. The annual incidence of TB is drastically decreased in Trieste after the closure of the Sanatorium, being now less than 10 new cases per 100,000 inhabitants, but the incidence in nineties was up to 30 new cases per 100,000 inhabitants. The fields of interest for the Pneumology Department of Trieste are tremendously increased in the last years changing location. To date, we have several cooperative investigations jointly with recognized scientific institutions of the Trieste's Area Science Park. Moreover, the Department of

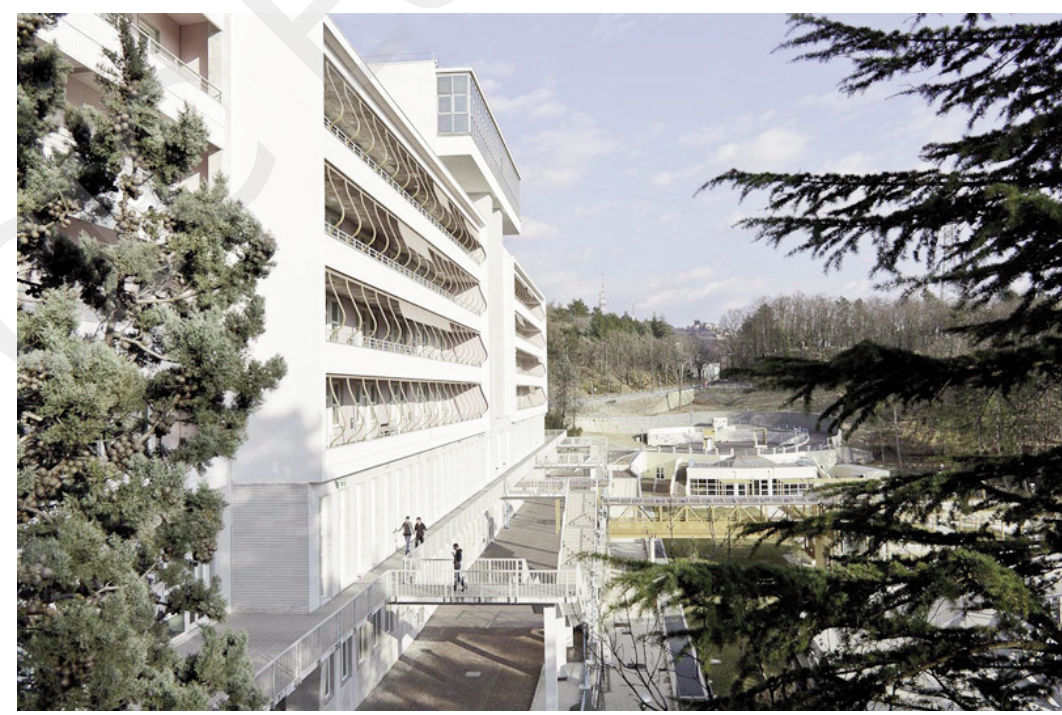

Figure 1 - The ex-sanatorium "Santorio Santorio", now restored location of the International School of Superior Studies, SISSA, at Trieste. 


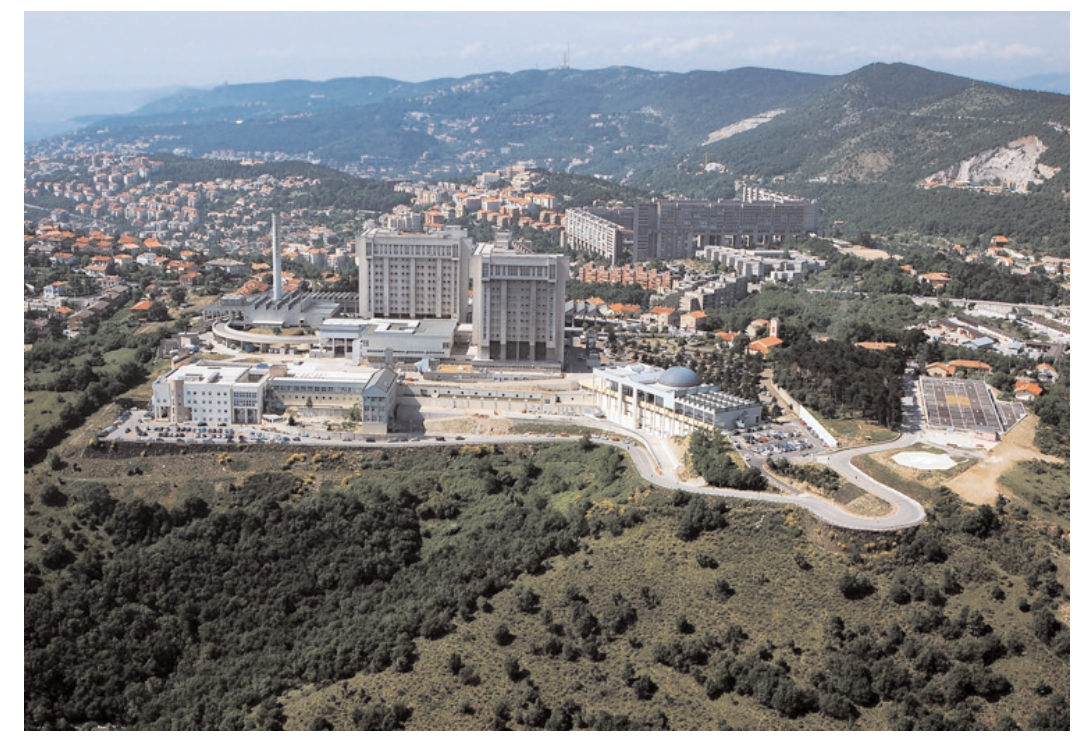

Figure 2 - The University Hospital of Cattinara, Trieste.

Respiratory Diseases at the University Hospital of Cattinara is now attracting patients from all regions of Italy, so loosing patient beds and changing hospital location doesn't mean worsening the medical offer for the patients, not at all. The medical concepts become obsolete faster and faster, and it is said today after less than every five years we should change half of our medical knowledge. Furthermore, also hospitals become old fast and they must be completely renewed after at least every $20-30$ years. This year is the thirtieth year since the opening of the Hospital Cattinara, and it was designed the restoration of the entire hospital by an Australian study of architecture with the construction of a Research Hospital for Children and Mothers in the same area of the Cattinara 's Hospital and linked to it (Figure 3). When we think to the redevelopment of a hospital it is important to intercept the medicine of the future, avoiding to think that a hospital is made only of bedrooms for the patients, with the usual support services (radiology, emergency room, operatory theatres). This is particularly important for an academic hospital, and for hospital with hub's characteristics. The present medicine needs high technology, but it also moving on towards molecular medicine, personalized medicine, predictive medicine, and regenerative medicine. Therefore an up-to-date renewed hospital must plan spaces dedicated to translational medicine (which are not only laboratories) and a general approach paperless with new forms of computer resources (grid network, bioinformatics facilities). A hospital is a permanent on site construction, but we need to re-think to it taking into account that medicine is becoming even more science than art, and new concepts are taking place today with a surprising rapidity.
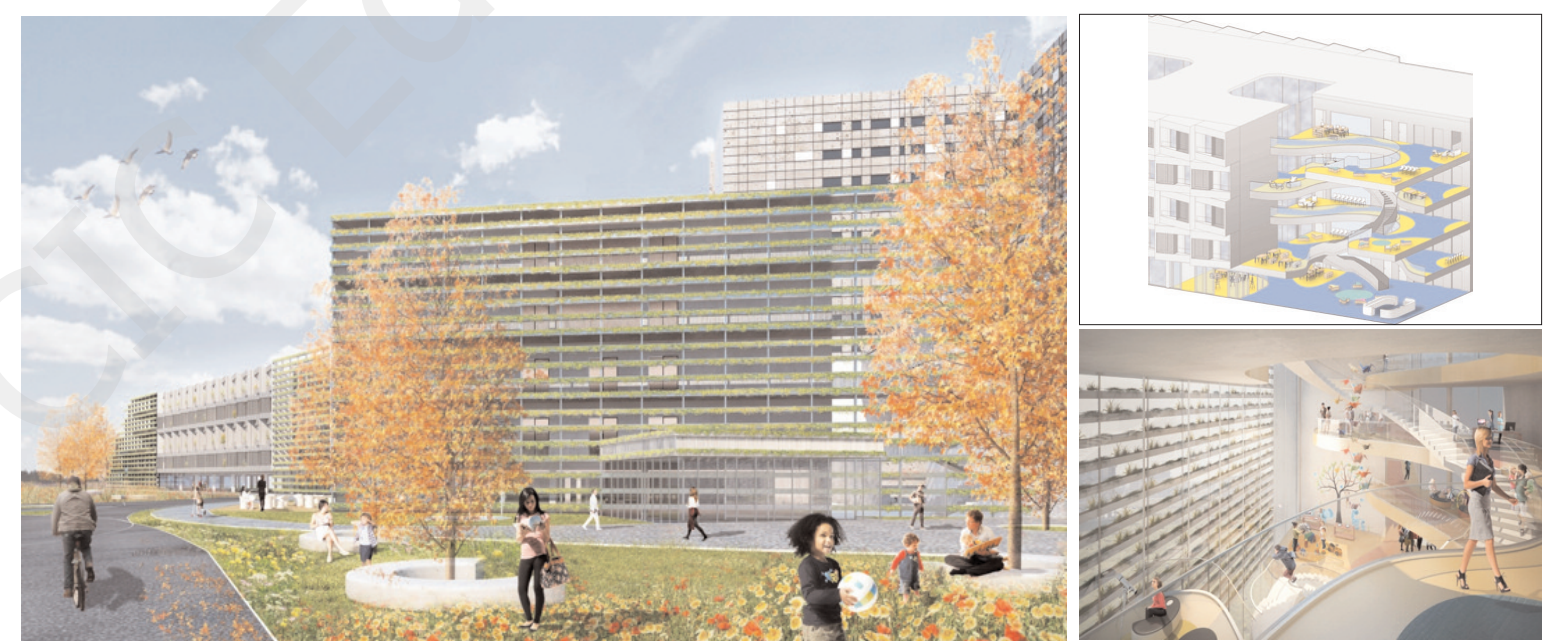

Figure 3 - Rendering of the redevelopment of the University Hospital of Cattinara, Trieste, Italy by BVN Donovan Hill, Australia. 